\title{
Expression of Wheat Gibberellins 2-oxidase Gene Induced Dwarf or Semi-dwarf Phenotype in Rice
}

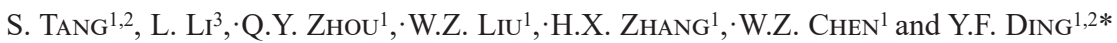 \\ ${ }^{1}$ College of Agronomy, Nanjing Agricultural University, Nanjing 210095, PR China \\ ${ }^{2}$ Jiangsu Collaborative Innovation Center for Modern Crop Production, Nanjing 210095, PR China \\ ${ }^{3}$ Editorial Department, Nanjing Agricultural University, Nanjing 210095, PR China
}

(Received 25 June 2018; Accepted 22 November 2018;

Communicated by A. Pécsváradi)

\begin{abstract}
Gibberellins (GAs) are a class of plant hormones that play important roles in diverse aspects during plant growth and development. A series of GA synthesis and metabolism genes have been reported or proved to have essential functions in different plant species, while a small number of GA 2-oxidase genes have been cloned or reported in wheat. Previous studies have provided some important findings on the process of GA biosynthesis and the enzymes involved in its related pathways. These may facilitate understanding of the complicated process underlying GA synthesis and metabolism in wheat. In this study, GA 2-oxidase genes TaGA2ox1-1, TaGA2ox1-2, TaGA2ox1-3, TaGA2ox1-4, TaGA2ox1-5, and TaGA2oxl-6 were identified and further overexpressed in rice plants to investigate their functions in GA biosynthesis and signaling pathway. Results showed overexpression of GA 2-oxidase genes in rice disrupted the GA metabolic pathways and induced catalytic responses and regulated other GA biosynthesis and signaling pathway genes, which further leading to GA signaling disorders and diversity in phenotypic changes in rice plants.
\end{abstract}

Keywords: gibberellins, gene expression, plant growth, rice, wheat

\section{Introduction}

Gibberellin (GA) belongs to a class of tetracyclic diterpenoids and plays a central role in regulating the process of plant growth and development. GA is synthesized from mevalonate by intermediates such as kauriene and transported upward from the xylem or transported non-polarly from the phloem down or bidirectional, and finally distributed mainly in plant roots, young leaves and seeds (Regnault et al. 2015; Binenbaum et al. 2018). The biosynthetic pathway of GA has been relatively clear and a great number of metabolic enzymes involved in GA synthesis have been identified, including ent-copalyl diphosphate synthase (CPS), ent-kaurene synthase (KS), ent-kaurene oxidase (KO), entkaurenoic acid oxidase (KAO), GA 20-oxidase (GA20ox), GA 3-oxidase(GA3ox), and GA 2-oxidase (GA2ox) (Chen et al. 2016). These proteins play a key regulatory role in coordinating GA synthesis and signal transduction and further regulate plant growth.

\footnotetext{
*Corresponding author; E-mail: dingyf@njau.edu.cn
} 
The biosynthesis of active GAs is a complex, multi-stepped process with diverse intermediates, among those a series of genes encoding enzymes related to GA biosynthesis have been identified (Figure S1*) (Hedden and Sponsel 2015; Pearce et al. 2015). For example, rice genes GA20ox, GA3ox, GA2ox and EUI1 (ELONGATEDUPPER INTERNODES1 catalyzed apparent oxidation) are primarily responsible for the regulation of GA biosynthesis and controls the elongation of internodes in wheat (Appleford et al. 2007). In wheat (Triticum aestivum), KS, KAO, GA20ox, GA3ox, GA2ox, RSG, and the negative regulator of RSG encoded genes were identified between a hybrid and the parents and were proved to have functions in controlling plant height and high endogenous GA levels (Zhang et al. 2007). Furthermore, Huang et al. (2012) cloned and functional analyzed of the upstream genes TaCPS, TaKS, TaKO, and TaKAO of the GA biosynthesis pathway and indicated the important roles of these genes and and their homologs during the growth and development process in wheat. In addition, the dwarfing genes $(R h t)$ that act as the transcriptional regulators in gibberellins signal transduction were identified and shown to be able to reduce the GA-mediated responses in wheat (Saville et al. 2012; Ford et al. 2018). However, only a few GA 2-oxidase genes have been cloned or reported in wheat and that makes it difficult to fully understand the complex process of GA synthesis and metabolism in wheat.

As the important enzyme involved in GA degradation, GA 2-oxidase hydroxylates the C-2 position of bioactive GAs such as GA1, GA4, and their immediate precursors GA20 and GA9 and transforms them into inactive GAs (Yamaguchi 2008). Studies based on physiological roles of GA 2-oxidases indicate that overexpression of genes encoding GA 2-oxidases can decrease GA levels in plants. In rice (Oryza sativa), ectopic expression of OsGA2ox1 produced a semi-dwarf phenotype (Sakamoto et al. 2003). Increased tiller numbers and adventitious root growth were also observed in GA 2-oxidases overexpressed rice mutants (Lo et al. 2008). Semi-dwarfism and tillering phenotypes are relevant for the cultivation of agronomic traits in crop breeding as semi-dwarf cultivars are more resistant to lodging and other environmental stresses and this may produce increased yields. In this study, GA 2-oxidase genes (following GenBank database accession numbers: TaGA2ox1-1 (JQ994299), TaGA2ox1-2 (JQ994300), TaGA2ox1-3 (JQ994301), TaGA2ox1-4 (JQ994302), TaGA2ox1-5 (JQ994303), and TaGA2ox1-6 (JQ994304)) were isolated and cloned from wheat and were further overexpressed in rice plants. Overexpression of GA 2-oxidase genes interrupted the process of rice growth and development and induced the significant GAs-deficient phenotype changes. These findings suggest the important biological functions of TaGA2oxl involved in the GA biosynthesis and signaling pathways and could provide a new insight into understanding the GA-mediated responses in cereal plants.

\section{Materials and Methods}

GA 2-oxidases cDNA fragments were isolated from the wheat cultivar "Chinese Spring" (Triticum aestivum). Seedlings were grown in plastic pots between $22^{\circ} \mathrm{C}$ and $25^{\circ} \mathrm{C}$ in the

*Further details about the Electronic Supplementary Material (ESM) can be found at the end of the article. 
environmental chamber to minimize the impact of environmental factors. Forty-day-old plants with similar height and body type were selected for the total RNA isolation experiment. Rice (Oryza sativa) cultivar "Nipponbare" was chosen as the transgenic model and rice seeds were surface sterilized in $2.5 \% \mathrm{NaClO}$ and placed on half-strength $\mathrm{Mu}-$ rashige and Skoog agar medium (Sigma-Aldrich) at $26{ }^{\circ} \mathrm{C}-28{ }^{\circ} \mathrm{C}$ for incubation. Transgenic plants were transferred to soil and grown in the experimental field at the research station of the Institute of Plant physiology \& Ecology, SIBS, CAS. Samples used for gene expression analysis were directly collected and lyophilized in liquid $\mathrm{N}_{2}$ for further molecular experiment.

\section{Identification and isolation of GA 2-oxidase encoded gene TaGA2ox1}

GA 2-oxidases gene sequence was identified from online databases: NCBI (www.ncbi. nlm.nih.gov/genbank/), TIGR database (http:/www.tigr.org/) and Rice Genome Annotation (RiceGAAS) database (http://ricegaas.dna.affrc.go.jp/). Total RNAs were isolated and purified from wheat leaves using the Trizol reagent (Invitrogen). cDNA libraries were constructed through reverse transcription using SuperScript ${ }^{\circledR}$ III First-Strand Synthesis System for RT-PCR (Invitrogen) and PCR enrichment. A putative GA 2-oxidase gene sequence from wheat (BT009225, full-length cDNA) was used for primer design using Primer 5.0. Primer pair (GA2ox1 sense primer 5'-GAGAGCATCTCCCCTGTTCC-3'; GA2ox1 anti-sense primer 5'-TGGTTCACACTAACTAACTATTCATCA-3') was performed for amplification using the Ex Taq ${ }^{\circledR}(\mathrm{TaKaRa})$ in DNA Thermal Cycler 480 with initial denaturing $\left(1 \mathrm{cycle}, 94^{\circ} \mathrm{C}, 3 \mathrm{~min}\right)$, denaturating $\left(35\right.$ cycles, $\left.94{ }^{\circ} \mathrm{C}, 1 \mathrm{~min}\right)$, primer annealing $\left(35\right.$ cycles, $\left.58^{\circ} \mathrm{C}, 30 \mathrm{~s}\right)$, primer extension $\left(35\right.$ cycles, $\left.72{ }^{\circ} \mathrm{C}, 90 \mathrm{~s}\right)$, final extension $\left(1 \mathrm{cycle}, 72{ }^{\circ} \mathrm{C}, 10 \mathrm{~min}\right)$ and holding at $4{ }^{\circ} \mathrm{C}$. Clones were randomly selected and sequenced from the wheat cultivar Chinese Spring using a primer design based on a wheat full-length cDNA sequence, a putative GA 2-oxidase gene in the public database (BT009225). For each clone, at least 1530 bp in length were sequenced using two-end sequencing. Unique sequences (at least one nucleotide differs from each other) with an intact ORF were produced. According to the phylogenetic analysis of translated amino acid sequences of the six sequences with other known GA 2-oxidase genes from other cereals, these sequences were grouped into one of two classes of this gene family (GA2ox1) (Figure S2). Those sequences were further ligated into the overexpression vector pUN1301 with maize ubiquitin promoter and transformed into rice variety Nipponbare for functional studies.

\section{Generation of overexpression constructs of TaGA2ox 1 and transformation}

Based on the sequencing results and alignment analysis, the overexpression constructs were created by PCR amplification using Ex Taq ${ }^{\circledR}$ (TaKaRa) and specific primers (sense 5'-GGTACCCTCTGCTTCTTTAG-3'; anti-sense 5'-GAGCTCACACGCACACGCAC-3') containing enzyme restriction sites (KpnI, Sacl) and for the GA2ox-encoding genes. PCR products were extracted and ligated to the pGEM®-T Easy Vector (Promega) 
for sequencing verification. Fragments obtained from correct amplifications were then digested with KpnI and SacI and ligated into the overexpression vector pUN1301 containing the maize ubiquitin promoter. Rice has been transformed through Agrobacterium mediated transfection with the use of mature embryo-derived calli. Protocols were based on the Hiei and Komari (2008) studies and transformation was performed with A. tumefaciens strain EHA105 harboring the plasmid pUN1301 and GUS was used as the reporter gene. Transformation efficiency was up to $70 \%$ and that was measured by counting the number of hygromycin-resistant calli or the number of transgenic plants.

\section{RT-PCR analysis}

Total RNA was isolated and purified from transgenic rice lines generated by overexpressing of TaGA2ox1-6 genes using the Trizol reagent (Invitrogen) and treated with RNasefree DNase I (Promega). cDNA libraries were constructed through reverse transcription with SuperScript ${ }^{\circledR}$ III First-Strand Synthesis System (Invitrogen). The synthesized cDNAs were served as templates for the following PCR analysis. Templates from transgenic rice and wild-type Nipponbare non-transformed control rice were amplified with Taq polymerase (Takara). Control primers Ubi sense (5'-TAAGCTGCCGATGTGCCTGCGTCG-3') and Ubi anti-sense (5'-CTGAAAGACAGAACATAATGAGCACAG-3') were used to amplify the ubiquitin (Ubi-1) transcripts. PCR conditions for the ubiquitin were $94{ }^{\circ} \mathrm{C}(30 \mathrm{~s}), 56{ }^{\circ} \mathrm{C}(30 \mathrm{~s})$, and $72{ }^{\circ} \mathrm{C}(90 \mathrm{~s})$ with 28 cycles and for the transgenic rice and controls, the cycles were adjusted to 35 cycles with primer-specific annealing temperatures. ACTIN was used as an endogenous control gene and all qRT-PCR primers (Table S1) were checked for efficiency and specificity by analyzing their amplification in a four fold dilution series and checking that each reaction yielded a single product by studying the derived dissociation curve.

\section{Sequence analysis}

A neighbor-joining phylogenetic tree was constructed using MEGA5.05 (http://www. megasoftware.net) for four protein sequences from the GA 2-oxidase genes (TaGA2ox1-1, TaGA2ox1-2, and TaGA2ox1-6 encode a same protein sequence) and their orthologs from other cereals (Tamura et al. 2011).

\section{Results}

\section{Overexpression of TaGA2ox genes in transgenic rice}

Total 6 overexpression vectors were constructed and recorded as TaGA2ox1-1 6. At seedling stage, all transgenic plants except TaGA2ox1-3 lines appeared dwarf phenotype with dark green leaf, stout roots (Fig. 1). Results showed the expression of GA20ox2 and GA3ox2 were induced in all transgenic plants, while GA3ox2 expression was undetectable in wild rice plants. Similarly, the expression of GA3ox2 was significantly lower in 
TaGA2ox1-3 overexpressed plants compared with other transgenic plants obtaining significant phenotypic changes. GID1 gene was down-regulated in all transgenic plants. However, the expression of the SLR1 gene showed no visible changes in both TaGA2oxl overexpressed and wild-type plants.

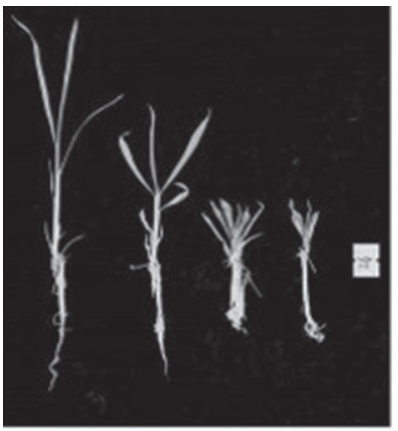

TaGA2Ox1-1 line

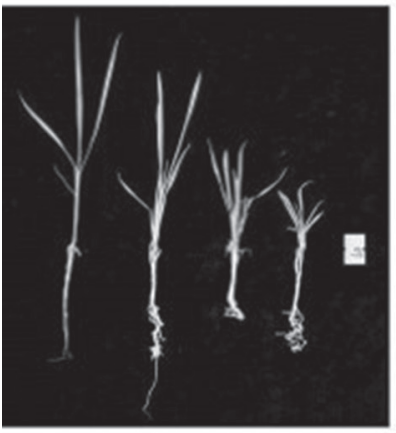

TaGA2ox1-4 line

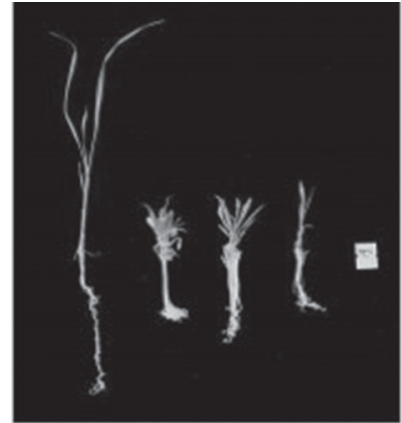

TaGA2Ox1-2 line

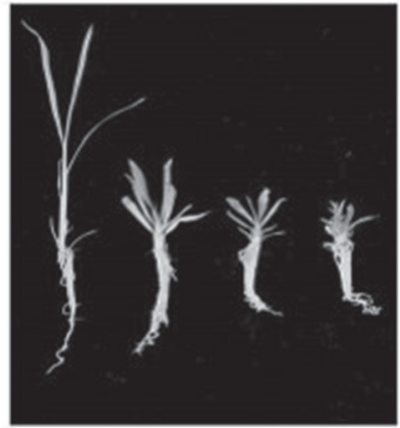

TaGA20x1-5 line

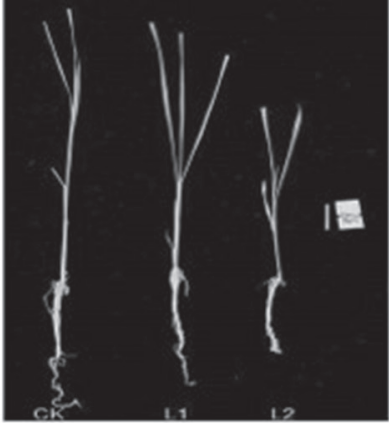

TaGA2ox1-3 line

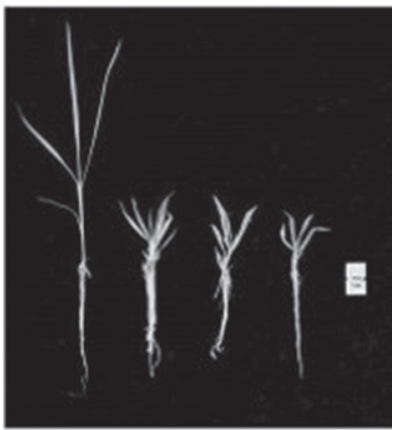

TaGA20x1-6 line

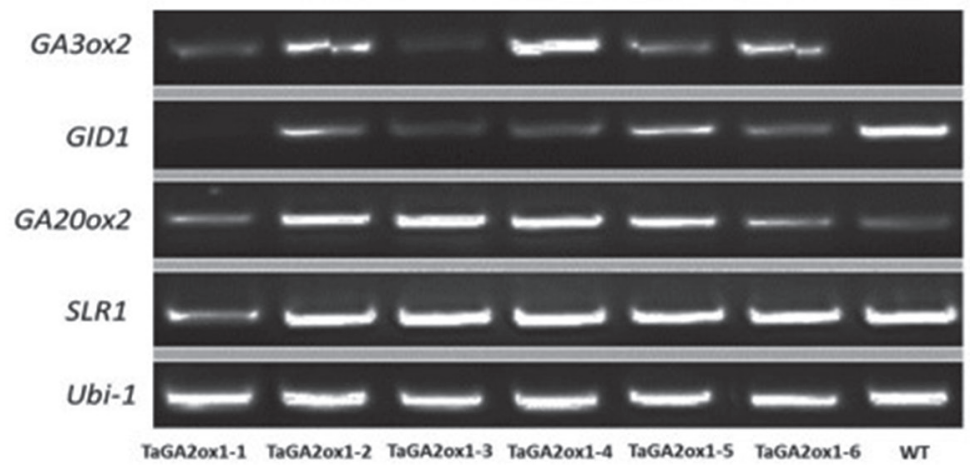

Figure 1. Phenotypic changes of TaGA2oxl overexpressed plants at seedling stage and RT-PCR analysis of the GA pathway genes in transgenic and wild-type plant seedlings 
Analysis of TaGA2ox1 gene transcripts

Results showed the TaGA2ox1-1, 2, 4, and 5 overexpressed plants were severely dwarfed, and the TaGA2ox1-6 overexpression transgenic plants showed slight dwarfism. TaGA2ox 1-3 overexpressed plants showed no visible differences in plant height compared with controls. Severe dwarf phenotype was obtained in TaGA2ox1-2 overexpressed plants and those plants can hardly develop any panicles. Different phenotypes were observed in TaGA2ox 1 overexpressed plants including slightly different from wild type to severely dwarfed with no discernible internodes (Fig. 2). Expressions of the TaGA2oxl genes were examined using RT-PCR and results indicated these TaGA2ox 1 encoded genes obtained different expression levels compared with the wild type. TaGA2oxl gene expression was up-regulated in all transgenic plants including TaGA2ox1-3 lines. Pheno-
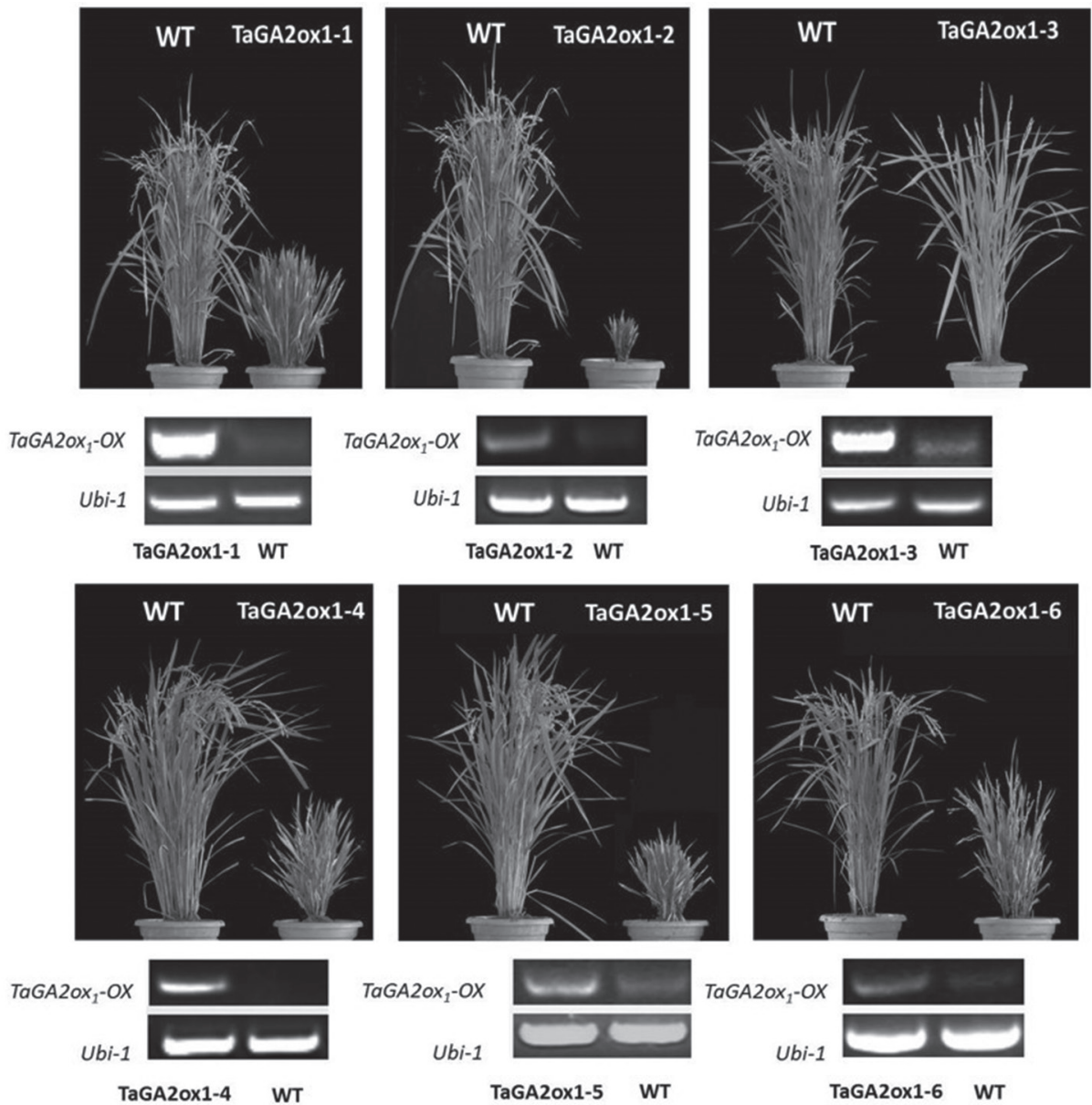

TaGA2ox $-O X$

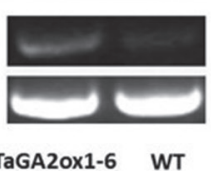

Figure 2. TaGA2ox1 overexpression on plant morphology 


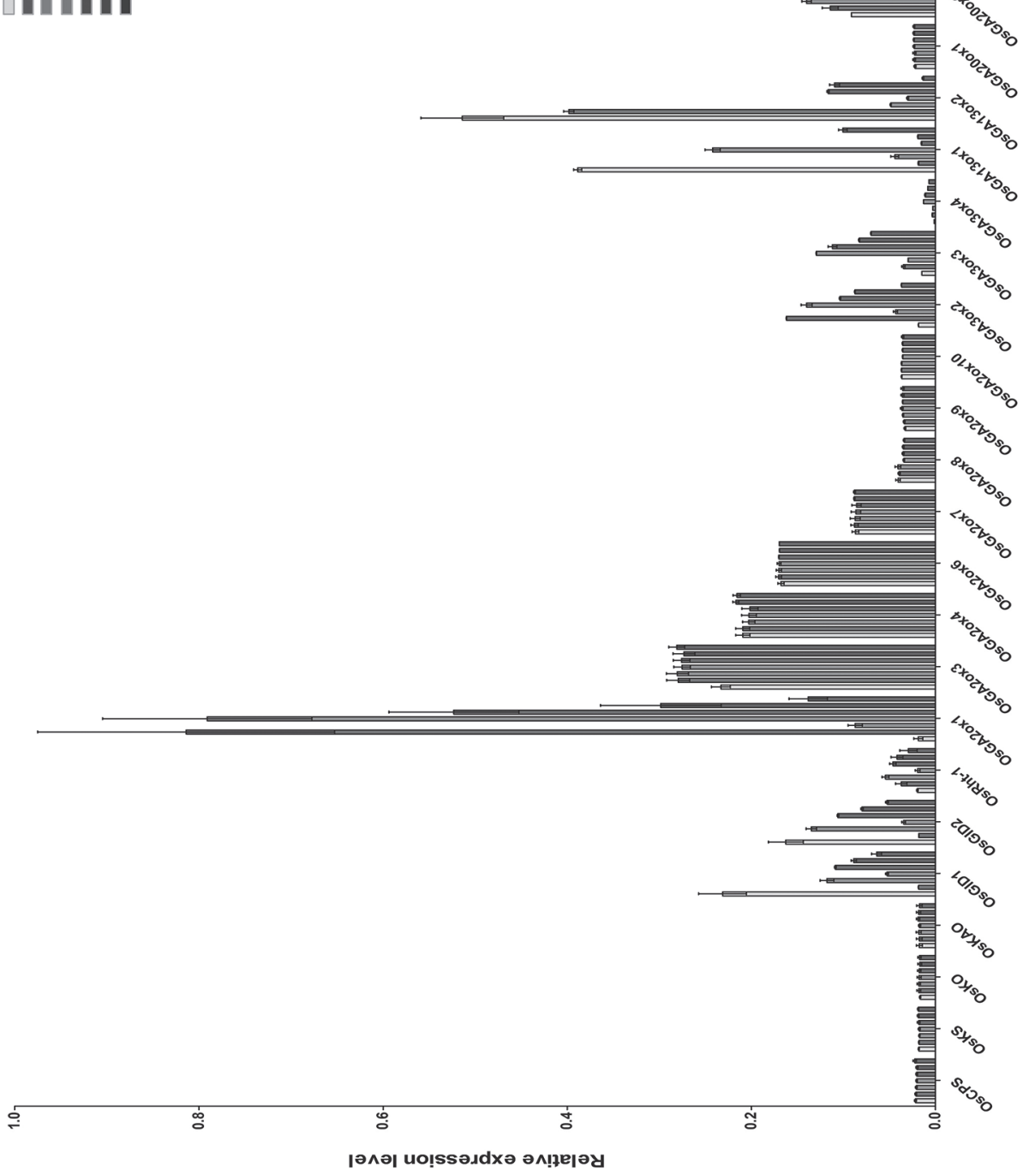


typic analysis showed that TaGA2ox1-3 overexpressed plants had no visible phenotype changes compared with controls. However, expression analysis showed TaGA2ox1 gene was up-regulated among all 3 replicates in TaGA2ox 1-3 lines and high levels of TaGA2ox 1 expression were also detected in all transgenic samples. Plant heights of all TaGA2oxl overexpressed rice lines obtained phenotype changes were significantly shorter than the wild type, and most of them cannot bare panicles (Figure S3). In transformed TaGA2ox1-3 rice lines, no obvious differences in phenotype from the non-transformed rice were observed and analysis of data from the survey confirmed this visual result.

\section{Expression analysis of GA biosynthesis and signaling pathway genes in TaGA2ox1 overexpressed rice plants}

GA biosynthetic and signaling genes were analyzed for their expression using qRT-PCR assays. Results indicated the tested GA pathway genes had different levels of expression, through either up-regulated or down-regulated, and some might have slight changes in expression and could not be detected here. There were some noticeable differences between expression levels of GA biosynthetic and signaling genes (Fig. 3). In general, the relative expression levels for GID1\&2 were significantly down-regulated in all TaGA2ox 1 overexpressed plants. OsGA13ox $1 \& 2$ genes were relatively down-regulated in TaGA2ox 1 transgenic lines. For those 2-oxoglutarate-dependent dioxygenases (2-ODDs: GA20ox, GA3ox, GA2ox), the expression levels of encoded genes were generally up-regulated. However, a small portion of GA pathway genes were detected to have no visible expression changes in wild-type and transgenic plants and some other genes, e.g. OsGA3ox 1 and $O s G A 20 x 5$ could not be detected in our plant samples.

\section{Discussion}

In this study, we investigated the GA 2-oxidase encoded genes through isolating and overexpressing the GA2ox1 encoded genes TaGA2ox1 in rice plants (Figure S4). Compared with wild type rice plants, the overexpression of GA 2-oxidase genes affected the processes of plant growth and development and further induced the GAs-deficiency phenotypes in plants. The phenotypic changes observed in this study could possibly be due to the overexpressed GA 2-oxidase genes stimulated the disorders of GA synthesis through affecting the catalytic activity of the GA 2-oxidases involved in GA biosynthesis and signaling pathways, which further interfered the synthesis and accumulation of GA levels in tested plants. Disorders of GA synthesis and accumulation could influence nearly every stage of plant development, e.g. root growth (Lo et al. 2008), internode elongation (Kaneko et al. 2003), and panicle initiation (Wu et al. 2016). In this case, significant phenotypic changes were observed in our study including dwarf at seedling and maturity stages, partial difficulty in panicle formation, etc. This may be due to overexpression of the GA 2-oxidase gene leading to the functional changes of important enzymes involved in GA pathway, which suggested the important roles of TaGA2ox1 in the regulation of plant development. 
In plants, bioactive GAs synthesis requires the catalyzing of GA20ox, GA3ox as well as GA2ox. GA2ox has been identified to have roles in catalyzing catabolism and inactivation of bioactive GAs or their precursors while GA20ox and GA3ox were supposed to active during the last few steps of GAs synthesis (Hedden and Sponsel 2015). In this study, we investigated the expression levels of those genes and results showed GA3ox2 was detected within all of the transformed rice obtained severe phenotypes and the expression level was significantly higher compared with the control. GA 20-oxidase activity is a major determinant of GA synthesis and signal levels in plants. GA20ox2 as the major GA20ox encoding gene expressed during most phases of vegetative and early reproductive development. In our results, GA20ox2 gene expression obtained similar up-regulated trend in transgenic plants and significantly lower expression was detected in non-transformed rice. The higher expression of GA20ox2 and GA3ox2 in our transformed lines may suggest that the GA-deficiency was induced by the overexpression of GA2oxl gene, which suppressed the synthesis of bioactive GAs at the upstream of the GAs pathway. In addition, GID, as a soluble receptor, mediates rice GA signaling and interacts with rice DELLA protein. Overexpression of GID1 resulted in a GA-hypersensitive phenotype and loss-of-function mutations could generate a pronounced dwarf phenotype with loss of GA responsiveness (Schwechheimer 2008; Hedden and Sponsel 2015). SLR, a DELLA protein in rice, is supposed to have functions in suppressing GA signaling by inducing various GA responses through degradation of those proteins. Generally, GIDI binds to SLR1 in a GA-dependent manner and works on the upstream of $S L R 1$ within the GA pathway. Altogether, those reported protein and genes have important functions in plant growth and development, especially in the GA signaling pathway. Our results demonstrated that GID1 and GID2 expressions were significantly down-regulated in TaGA2ox1 overexpressed rice lines compared with wild type. SLR1 gene showed no apparent expression changes either in transgenic rice or controls, which would probably due to the GA signal blocking induced by the down-regulation of GID encoded genes in TaGA2ox 1 overexpressed rice line. However, we did not detect the expression of these genes in rice samples, probably due to the expression site specificity according to Pearce and colleagues' conclusion, some GA related genes were predominantly expressed in developing grain, e.g. GA1ox1, GA3oxl (Pearce et al. 2015). In our case, most of the TaGA2oxl overexpressed rice lines obtained apparently dwarf phenotypes and could not develop any panicles.

In summary, overexpression of the GA 2-oxidases encoded genes in rice induced a GA-deficiency phenotype, indicating the biological functions of TaGA2ox 1 genes in rice growth and development process. Overexpression of TaGA2oxl induced catalytic responses and regulated other GA synthesis and signaling pathway genes, further leading to GA signaling disorders in overexpressing plants, and that eventually generated the phenotypic changes in rice plants.

\section{Accession numbers}

Sequence data from this article can be found in the GenBank databases under the following accession numbers: TaGA2ox1-1 (JQ994299), TaGA2ox1-2 (JQ994300), 
TaGA2ox1-3 (JQ994301), TaGA2ox1-4 (JQ994302), TaGA2ox1-5 (JQ994303), and TaGA2ox1-6 (JQ994304).

\section{Acknowledgements}

This study was jointly funded by the National Key R\&D Program of China (2017YFD0300100), the National Natural Science Foundation of China (31701366), Jiangsu Collaborative Innovation Center for Modern Crop Production (JCIC-MCP) and the Priority Academic Program Development of Jiangsu Higher Education Institutions (PAPD). We would like to thank Dr. Kang Chong (Institute of Botany, The Chinese Academy of Sciences) for providing the overexpression vector pUN1301 containing the maize ubiquitin promoter. The authors declare that they have no conflict of interest.

\section{References}

Appleford, N.E., Wilkinson, M.D., Ma, Q., Evans, D.J., Stone, M.C., Pearce, S.P., Lenton, J.R. et al. 2007. Decreased shoot stature and grain alpha-amylase activity following ectopic expression of a gibberellin 2-oxidase gene in transgenic wheat. J. Exp. Bot. 58:3213-3226.

Binenbaum, J., Weinstain, R., Shani, E. et al. 2018. Gibberellin localization and transport in plants. Trends Plant Sci. 23:410-421.

Chen, J.J., Xie, J.H., Duan, Y.J., Hu, H.G., Hu, Y.L., Li, W.M. 2016. Genome-wide identification and expression profiling reveal tissue-specific expression and differentially-regulated genes involved in gibberellin metabolism between Williams banana and its dwarf mutant. BMC Plant Biol. 16:123.

Ford, B., Foo, E., Sharwood, R.E., Karafiatova, M., Vrána, J., MacMillan, C., Spielmeyer, W. et al. 2018. Rht18 Semi-dwarfism in wheat is due to increased expression of GA 2-oxidaseA9 and lower GA content. Plant Physiol. 177:168-180.

Hedden, P., Sponsel, V. 2015. A century of gibberellin research. J. Plant Growth Regul. 34:740-760.

Hiei, Y., Komari, T. 2008. Agrobacterium-mediated transformation of rice using immature embryos or calli induced from mature seed. Nat. Protoc. 3:824-834.

Huang, Y., Yang, W., Pei, Z., Guo, X., Liu, D., Sun, J., Zhang, A. 2012. The genes for gibberellin biosynthesis in wheat. Funct. Integr. Genomics 12:199-206.

Kaneko, M., Itoh, H., Inukai, Y., Sakamoto, T., Ueguchi-Tanaka, M., Ashikari, M., Matsuoka, M. 2003. Where do gibberellin biosynthesis and gibberellin signaling occur in rice plants? Plant J. 35:104-115.

Lo, S.F., Yang, S.Y., Chen, K.T., Hsing, Y.I., Zeevaart, J.A., Chen, L.J., Yu, S.M. 2008. A novel class of gibberellin 2-oxidases control semidwarfism, tillering, and root development in rice. Plant Cell. 20:2603-2618.

Pearce, S., Huttly, A.K., Prosser, I.M., Li, Y.D., Vaughan, S.P., Gallova, B., Phillips, A.L. et al. 2015. Heterologous expression and transcript analysis of gibberellin biosynthetic genes of grasses reveals novel functionality in the GA3ox family. BMC Plant Biol. 15:130.

Regnault, T., Davière, J.-M., Wild, M., Sakvarelidze-Achard, L., Heintz, D., Carrera Bergua, E., Achard, P. et al. 2015. The gibberellin precursor GA12 acts as a long-distance growth signal in Arabidopsis. Nat. Plants. 1:15073.

Sakamoto, T., Morinaka, Y., Ishiyama, K., Kobayashi, M., Itoh, H., Kayano, T., Tanaka, H. et al. 2003. Genetic manipulation of gibberellin metabolism in transgenic rice. Nat. Biotechnol. 21:909-913.

Saville, R.J., Gosman, N., Burt, C.J., Makepeace, J., Steed, A., Corbitt, M., Nicholson, P. et al. 2012. The 'Green Revolution' dwarfing genes play a role in disease resistance in Triticum aestivum and Hordeum vulgare. J. Exp. Bot. 63:1271-1283.

Schwechheimer, C. 2008. Understanding gibberellic acid signaling-are we there yet? Curr. Opin. Plant Biol. 11:9-15. 
Wu, Y., Wang, Y., Mi, X.F., Shan, J.X., Li, X.M., Xu, J.L., Lin, H.X. 2016. The QTL GNP1 encodes GA20ox1, which increases grain number and yield by increasing cytokinin activity in rice panicle meristems. PLoS Genet. 12:e1006386

Yamaguchi, S. 2008. Gibberellin metabolism and its regulation. Annu. Rev. Plant Biol. 59:225-251.

Zhang, Y., Ni, Z., Yao, Y., Nie, X., Sun, Q. 2007. Gibberellins and heterosis of plant height in wheat (Triticum aestivum L.). BMC Genet. 8:1-10.

\section{Electronic Supplementary Material (ESM)}

Electronic Supplementary Material (ESM) associated with this article can be found at the website of CRC at https://akademiai.com/loi/0806

Electronic Supplementary Figure S1. Illustration of gibberellin (GA) biosynthetic pathway

Electronic Supplementary Figure S2. Phylogenetic relationship of gibberellin 2-oxidase genes from wheat and other cereals based on their amino acid sequences

Electronic Supplementary Figure S3. Comparison of the plant heights of Nipponbare and TaGA2ox1 overexpressed rice plants. Bars represent the means \pm SD of at least 10 different seedlings. Significant differences between Nipponbare and TaGA2oxl transgenic rice were tested using one-way ANOVA to determine the significance of the differences. $*$ and $* *$ indicate significant differences at $* * P \leq 0.001$ and $* P \leq 0.01$

Electronic Supplementary Figure S4. Nucleotide sequence alignment of GA2oxs in rice

Electronic Supplementary Table S1. Primer sequences used for RT-PCR in this study 